\title{
Mid-IR observations of small stellar clusters surrounding Herbig AeBe stars ${ }^{\star}$
}

\author{
E. Habart ${ }^{1}$, L Testi $^{1}$, A. Natta ${ }^{1}$, and L. Vanzi ${ }^{2}$ \\ 1 Osservatorio Astrofisico di Arcetri, INAF, Largo E. Fermi 5, 50125 Firenze, Italy \\ 2 European Southern Observatory, Santiago, Chile
}

Received 23 September 2002 / Accepted 28 November 2002

\begin{abstract}
We present TIMMI 2 observations at $10 \mu \mathrm{m}$ of 12 fields centered on known Herbig AeBe (HAeBe) stars. We detected sources (in addition to the HAeBe star) in five fields, three of which are associated with early B stars. Using complementary near-IR and optical data, we estimate that the detected sources are young, embedded $\left(A_{V} \sim 5-24\right.$ mag), intermediate-mass objects $\left(\sim 1.5-3.5 M_{\odot}\right)$. In four objects the $10 \mu \mathrm{m}$ emission can be accounted for by a circumstellar disk, while in 2 cases (possibly 3) there is evidence that the objects are still in an earlier stage of evolution (Class I). These results confirm the association of the early B Herbig stars with rather rich clusters, as well as their very young age. If our estimates of the stellar parameters are confirmed, this would be the largest sample so far of embedded, intermediate-mass objects available for further studies.
\end{abstract}

Key words. stars: formation - stars: pre-main sequence - infrared: stars - open clusters and associations: general - circumstellar matter

\section{Introduction}

Small clusters of stars associated with pre-main-sequence (PMS) stars of intermediate mass have been detected by Testi et al. (1998), Testi et al. (2002). These authors found a statistically significant enhancement of the number of objects around Herbig Be/Ae stars (HAeBe) in $K$-band images. They also found that the number of companions was a steep function of the mass of the $\mathrm{HAe} / \mathrm{Be}$ star, being very small or zero for stars with masses lower than about $5 M_{\odot}$, and of the order of 10-70 objects within a radius of $0.2-0.4$ pc for B0 stars (mass about $\left.15 M_{\odot}\right)$.

This paper presents the first results of a more detailed study of the properties of such clusters. In particular, we have obtained 10 micron images of a subset (12 regions) of the fields studied by Testi et al. (1998), Testi et al. (2002), with the aim of detecting excess emission, possibly due to circumstellar disks, associated with the companions of the HAeBe star. For most of the detected objects, we have also obtained near-infrared broad-band photometry and, when possible, optical magnitudes and spectra, that allows us to assign spectral types and to locate the stars on the HR diagram. In this way, we were planning to determine the age of the companions, providing a strong

Send offprint requests to: E. Habart,

e-mail: habart@arcetri.astro.it

* Based on observations collected at the European Southern Observatory, La Silla, Chile, ESO proposal numbers 65.I-0097, 66.C-0064, 66.C-0120, 67.C-0041, part of the data were acquired using the ESO Service Mode. constraint on the age of the Herbig Be star itself, which is very poorly known. Also, we were interested in getting information on the fraction of stars with/without disks in these small clusters. The first results discussed in this paper are encouraging, and show that our strategy can indeed provide interesting information. However, it is clear that one needs additional and more sensitive observations, both at 10 microns and in the near-IR and optical, before firm conclusions can be reached.

The paper is organized as follows. Section 2 describes the selected fields; Sect. 3 presents the observations and the data reduction; the results are discussed in Sect. 4, Sect. 5 (where we derive the properties, extinction and mass in particular, of each individual $10 \mu \mathrm{m}$ source we detected), and Sect. 6. A summary follows in Sect. 7.

\section{Sources}

We have selected 12 fields around well-known HAeBe stars from the near IR surveys of Testi et al. (1998), Testi et al. (2002). Table 1 lists the names of the HAeBe stars, their spectral types, distances, effective temperatures, luminosities, extinctions, and ages. The distances are from Testi et al. (1999), Testi et al. (2002) and references therein. Effective temperatures, extinctions and luminosities have been determined from the broad-band $V$ magnitudes, $(B-V)$ and spectral types, as described in Testi et al. (1998). The ages have been obtained by comparing the location of each star on the HR diagram with Palla \& Stahler (1993) evolutionary tracks. For each star we also report in Table 1 the number of expected companions 
based on the near infrared surveys results (Testi et al. 1998; Testi et al. 2002), which, in most fields, are expected to be sensitive down and below $0.1 M_{\odot}$.

Note that for each field our $10 \mu \mathrm{m}$ images cover the entire extent of the near-IR cluster in most cases, except for a few nearby objects. The values of $N_{\mathrm{m}}$ given in Table 1 have been computed for the area of each cluster covered by our $10 \mu \mathrm{m}$ images.

\section{Observations and data reduction}

\subsection{TIMMI2 observations}

Mid-IR observations of the 12 fields were obtained at the ESO $3.6 \mathrm{~m}$ telescope with the mid-infrared spectrograph/imager TIMMI2 (Thermal Infrared Multimode Instrument), equipped with a $240 \times 320 \mathrm{Si}$ :As array detector. Imaging observations have been obtained with a pixel size of $0.2^{\prime \prime} /$ pixel and $0.3^{\prime \prime} /$ pixel in the narrow band filters $\mathrm{N} 2\left(\lambda_{\mathrm{c}}=10.53 \mu \mathrm{m}\right.$, $\Delta \lambda=1.46 \mu \mathrm{m})$ and $\mathrm{N} 11.9\left(\lambda_{\mathrm{c}}=11.55 \mu \mathrm{m}, \Delta \lambda=1.13 \mu \mathrm{m}\right)$ over two nights in June 2001 with a seeing of $\sim 1^{\prime \prime}$. The area covered by each image is about $1 \mathrm{sq}$ arcmin, adequate to cover the typical cluster extent. For $\sim 1$ hour on-source integration time, the sensitivity was $0.05 \mathrm{Jy}(3 \sigma)$. To subtract the atmospheric contamination the $10 \mu \mathrm{m}$ data were acquired doing chopping and nodding: nodding is done perpendicular to the chopping direction (chop- and nod-angles are such that the source appears once in each quadrant of the array). Then, the chop- and nod- images were aligned and co-added.

The photometric calibration of some of the fields turned out to be rather poor. During our observing run, we observed a set of ESO mid-IR photometric standard star. However, not all the nights were of photometric quality, and the N11.9 standards in particular showed a large dispersion (about a factor 2). We have therefore used existing ISO/SWS observations of 5 of the Herbig star in our list (MWC 297, V921 Sco, MWC 614, VV Ser, HD 97048, van Kerckhoven et al. 2000; Siebenmorgen et al. 2000) to check the calibration. We found that for the filter $\mathrm{N} 2$ the discrepancy was of $25 \%$ at most, comparable to the dispersion among our standards. For the N11.9 filter, on the other hand, the ISO fluxes were about $50 \%$ higher than what we derive, again within the dispersion of the measurements of the standard stars. We have then adopted the ISO/SWS calibration and attributed a calibration uncertainty of $25 \%$ to measurements in the N2 filter and of 50\% to those in the N11.9 filter.

The measured fluxes are reported in Table 2. Note that for the brightest objects (fluxes $\gtrsim 0.1 \mathrm{Jy}$ ), the largest contribution to the final photometric uncertainties come from the calibration uncertainties just discussed. Very weak objects in the table (fluxes $<0.1 \mathrm{Jy}$ ) are typically a 3-4 sigma detections.

\subsection{Near-IR photometry and optical spectra}

The $10 \mu \mathrm{m}$ data have been complemented by a number of observations at shorter wavelengths. Near infrared photometry for the TIMMI2 companions of V921 Sco and LSS 3027B was obtained with SOFI at the ESO-NTT either from a deep survey of about 30 fields around southern HBe stars
(Testi et al. 2002) or from dedicated short exposure observations carried-on in March 2001. The data were reduced and calibrated using standard procedures and observations of standard stars from the lists of Hunt et al. (1998) and Persson et al. (1998). Calibration uncertainties are about 5\%. For the companions of VY Mon, near-IR magnitudes have been published by Testi et al. (1998).

Optical photometry and low-resolution spectroscopy for companions in the VY Mon and LSS 3027B fields were obtained at the ESO-Danish $1.5 \mathrm{~m}$ telescope with the DFOSC instrument in May and September 2000 and January and March 2001. Photometric calibration was ensured by observations of standard stars from the Landolt (1992) list. The Gunn- $i$ magnitudes of the Landolt standards were computed using the transformations in Fukugita et al. (1996), and are presented here in the AB magnitude scale. The spectroscopic observations were performed using the low-resolution DFOSC red grism (GR5), Hamuy et al. (1994) standards were used for response calibration.

The two $10 \mu \mathrm{m}$ companions of HD 97048 are known mid-IR emitters (Persi et al. 2000), called HM 16 (also ISOChaI-119) and ISO-ChaI-126, respectively. $I, J$, and $K_{\mathrm{s}}$ near infrared magnitudes can be found in the ISOCAM-DENIS survey of Persi et al. (2000). $V$-band photometry and $\mathrm{H} \alpha$ equivalent width of HM 16 (61 ̊) are from Comerón et al. (1999), who assign a spectral type $\mathrm{K} 7$ to this source.

The two companions of MWC 297, to the best of our knowledge, have no measured fluxes at shorter wavelengths. They were too bright in the near-IR for the observations of Testi et al. (1998).

A summary of the available photometric points and of the $\mathrm{H} \alpha$ equivalent widths is reported in Table 3 for the $10 \mu \mathrm{m}$ companions of V 921 Sco, LSS 3027B and VY Mon. The observed spectral energy distributions (SEDs) are shown in Fig. 1.

The optical spectra of the two infrared sources in the VY Mon and LSS 3027 B fields are shown in Fig. 2. The S/N of the latter spectrum does not allow us to detect any absorption or emission line besides the strong H $\alpha$. The VY Mon-\#1 spectrum has a higher signal to noise and, in addition to $\mathrm{H} \alpha$ and [OI] $\lambda 6300$ emission, we detect several photospheric lines. The most prominent are: the Na I $\lambda 5890 \lambda 5896$ doublet, the Ca I $\lambda 6162$, and the Fe I, Ca I, Ba II blend at $\lambda 6497$. We tentatively detect the $\mathrm{Li}$ I $\lambda 6708$ line with equivalent width $-0.4 \pm 0.1$.

\section{Results}

The number of $10 \mu \mathrm{m}$ companions for each $\mathrm{HAeBe}$ star is given in the last column of Table 1. This number is never large, and in many fields we do not detect any object in addition to the Herbig star itself. This is the result of a combination of effects. First of all, only the more massive HAeBe stars are found in relatively rich clusters, and in fact 3 over 5 of the fields with companions contain a star earlier than B1. Conversely, 6 out of 7 of the empty fields are associated to stars later than B5. The exceptions are VY Mon, a B8 star with a relatively rich near-IR cluster (Testi et al. 1998) and HD 97048, a nearby A0 star in ChaI. 
Table 1. HAeBe stars.

\begin{tabular}{lcccccccc}
\hline \hline Source & ST & $\begin{array}{c}d \\
(\mathrm{pc})\end{array}$ & $\begin{array}{c}\mathrm{Lg} T_{\text {eff }} \\
(\mathrm{K})\end{array}$ & $\begin{array}{c}\mathrm{Lg} L_{\star} \\
\left(L_{\odot}\right)\end{array}$ & $\begin{array}{c}A_{V} \\
(\mathrm{mag})\end{array}$ & $\begin{array}{c}\text { Age } \\
(\mathrm{Myr})\end{array}$ & $N_{\mathrm{m}}$ & $\begin{array}{c}10 \mu \mathrm{m} \\
\text { companions }\end{array}$ \\
\hline MWC 297 & O9 & 450 & 4.52 & 4.7 & 7.9 & $0.1-1$ & 20 & 2 \\
V921 Sco & B0 & 800 & 4.49 & 4.3 & 5.0 & $0.1-1$ & 40 & 4 \\
LSS 3027 & B1 & 1800 & 4.35 & 3.6 & 1.7 & $0.1-1$ & 15 & 2 \\
LkHa 119 & B1 & 1400 & 4.35 & 3.5 & 3.3 & $0.1-1$ & 2 & - \\
Hen3-847 & B5 & 630 & 4.14 & 1.9 & 0.7 & $>1$ & 5 & - \\
HD 17081 & B7 & 135 & 4.09 & 2.7 & 0.0 & 0.1 & 3 & - \\
VY Mon & B8 & 800 & 4.05 & 2.8 & 5.3 & 0.1 & 23 & 2 \\
MWC 614 & B9 & 240 & 4.03 & 2.7 & 1.8 & 0.1 & 5 & - \\
HD 149914 & B9 & 165 & 4.03 & 2.2 & 0.9 & $0.5-1$ & 4 & - \\
VV Ser & B9 & 440 & 4.03 & 2.0 & 3.2 & 1 & 17 & - \\
HD 97048 & A0 & 160 & 3.99 & 1.5 & 1.0 & $\geq 5$ & 3 & 2 \\
TY CrA & A5 & 130 & 3.92 & 0.9 & 1.2 & $\geq 10$ & 6 & - \\
\hline
\end{tabular}

Table 2. Mid-IR photometry.

\begin{tabular}{|c|c|c|c|c|}
\hline Source & $\alpha_{2000}$ & $\delta_{2000}$ & $\begin{array}{l}N 2 \\
(\mathrm{Jy})\end{array}$ & $\begin{array}{c}N 11.9 \\
(\mathrm{Jy})\end{array}$ \\
\hline MWC297 & $18: 27: 39.6$ & $-03: 49: 52$ & 82 & - \\
\hline \#1 & $\alpha_{0}+36^{\prime \prime}$ & $\delta_{0}+14^{\prime \prime}$ & 0.42 & - \\
\hline \#2 & $\alpha_{0}+15^{\prime \prime}$ & $\delta_{0}-19^{\prime \prime}$ & 0.34 & - \\
\hline V921 Sco & $16: 55: 33.8$ & $-42: 42: 08$ & 40 & 46.4 \\
\hline \#1 & $\alpha_{0}-13^{\prime \prime}$ & $\delta_{0}+6^{\prime \prime}$ & 1.35 & 0.6 \\
\hline \#2 & $\alpha_{0}+1^{\prime \prime}$ & $\delta_{0}+5^{\prime \prime}$ & 0.11 & 0.04 \\
\hline \#3 & $\alpha_{0}+6^{\prime \prime}$ & $\delta_{0}+6^{\prime \prime}$ & 0.07 & 0.06 \\
\hline \#4 & $\alpha_{0}+5^{\prime \prime}$ & $\delta_{0}-10^{\prime \prime}$ & 0.09 & 0.07 \\
\hline LSS 3027B & 13:19:04 & $-62: 34: 10$ & 0.95 & - \\
\hline \#1 & $\alpha_{0}-8^{\prime \prime}$ & $\delta_{0}+0.5^{\prime \prime}$ & 0.9 & - \\
\hline VY-Mon & $06: 31: 6.9$ & $+10: 26: 05$ & - & 34.2 \\
\hline \#1 & $\alpha_{0}-32.5^{\prime \prime}$ & $\delta_{0}+6^{\prime \prime}$ & - & 0.05 \\
\hline \#2 & $\alpha_{0}-14.5^{\prime \prime}$ & $\delta_{0}-16^{\prime \prime}$ & - & 0.05 \\
\hline HD 97048 & 11:08:03.3 & $-77: 39: 17.5$ & 5.4 & - \\
\hline$\# 1$ & $\alpha_{0}+17^{\prime \prime}$ & $\delta_{0}+33^{\prime \prime}$ & 4.5 & - \\
\hline $\begin{array}{l}\text { (HM 16) } \\
\# 2 \\
\text { (ISO-ChaI-126) }\end{array}$ & $\alpha_{0}+1^{\prime \prime}$ & $\delta_{0}+35^{\prime \prime}$ & 0.7 & - \\
\hline LkHa 119 & $18: 05: 56$ & $-24: 14.7$ & 1.5 & - \\
\hline Hen3-847 & 13:01:17.8 & $-48: 53: 18.8$ & 36.7 & - \\
\hline HD 17081 & 02:44:07.3 & $-13: 51: 31.3$ & 0.222 & - \\
\hline MWC 614 & 19:11:11.2 & $+15: 47: 15.6$ & 27.4 & - \\
\hline HD 149914 & $16: 38: 28.6$ & $-18: 13: 13.7$ & 0.075 & - \\
\hline VV Ser & $18: 28: 49$ & $+00: 08: 39$ & 4.3 & - \\
\hline TY Cra & 19:01:40.8 & $-36: 52: 33.9$ & 1.12 & - \\
\hline
\end{tabular}

The distance of the region is another obvious factor that limits the detectability of sources. Let us assume, for simplicity, that all the companions of the HAeBe star have circumstellar disks heated by the stellar radiation, and that at $10 \mu \mathrm{m}$ one detects the disk emission. If the age of the star (and therefore its luminosity) are known, it is then possible to obtain a rough estimate of the minimum mass of the star whose disk emission one can detect (for our $3 \sigma$ limit of $0.05 \mathrm{Jy}$ ) as function of distance (Fig. 3). For ages in the interval 0.1-1 Myr, we find that low-mass objects are detectable only in nearby regions. 
Table 3. Optical and near-infrared photometry.

\begin{tabular}{|c|c|c|c|c|c|c|c|}
\hline Sources & $\begin{array}{c}V \\
\text { (mag) }\end{array}$ & $\begin{array}{c}R \\
(\mathrm{mag})\end{array}$ & $\begin{array}{c}i \\
(\mathrm{mag})\end{array}$ & $\begin{array}{c}J \\
(\mathrm{mag})\end{array}$ & $\begin{array}{c}H \\
(\mathrm{mag})\end{array}$ & $\begin{array}{c}K \mathrm{~s} \\
(\mathrm{mag})\end{array}$ & $\begin{array}{l}\mathrm{H} \alpha \\
(\AA)\end{array}$ \\
\hline \multicolumn{8}{|c|}{ V921 Sco field } \\
\hline$\# 1$ & - & - & - & $13.16 \pm 0.03$ & $11.25 \pm 0.01$ & $9.82 \pm 0.01$ & - \\
\hline$\# 2$ & - & - & - & $11.97 \pm 0.01$ & $10.59 \pm 0.01$ & $9.46 \pm 0.01$ & - \\
\hline \#3 & - & - & - & $>15$ & $13.53 \pm 0.06$ & $11.59 \pm 0.03$ & - \\
\hline \#4 & - & - & - & $15.55 \pm 0.13$ & $12.16 \pm 0.02$ & $10.06 \pm 0.01$ & - \\
\hline \multicolumn{8}{|c|}{ LSS 3027B field } \\
\hline$\# 1$ & - & - & $19.14 \pm 0.07$ & $14.92 \pm 0.01$ & $12.55 \pm 0.01$ & $10.94 \pm 0.01$ & 39 \\
\hline \multicolumn{8}{|c|}{ VY Mon field } \\
\hline$\# 1$ & $16.71 \pm 0.05$ & $15.42 \pm 0.05$ & $14.6 \pm 0.2$ & $12.26 \pm 0.01$ & $10.83 \pm 0.01$ & $9.71 \pm 0.01$ & 32 \\
\hline$\# 2$ & $>21.5$ & $>20.5$ & $19.9 \pm 0.1$ & $16.25 \pm 0.06$ & $13.79 \pm 0.02$ & $11.65 \pm 0.01$ & - \\
\hline
\end{tabular}

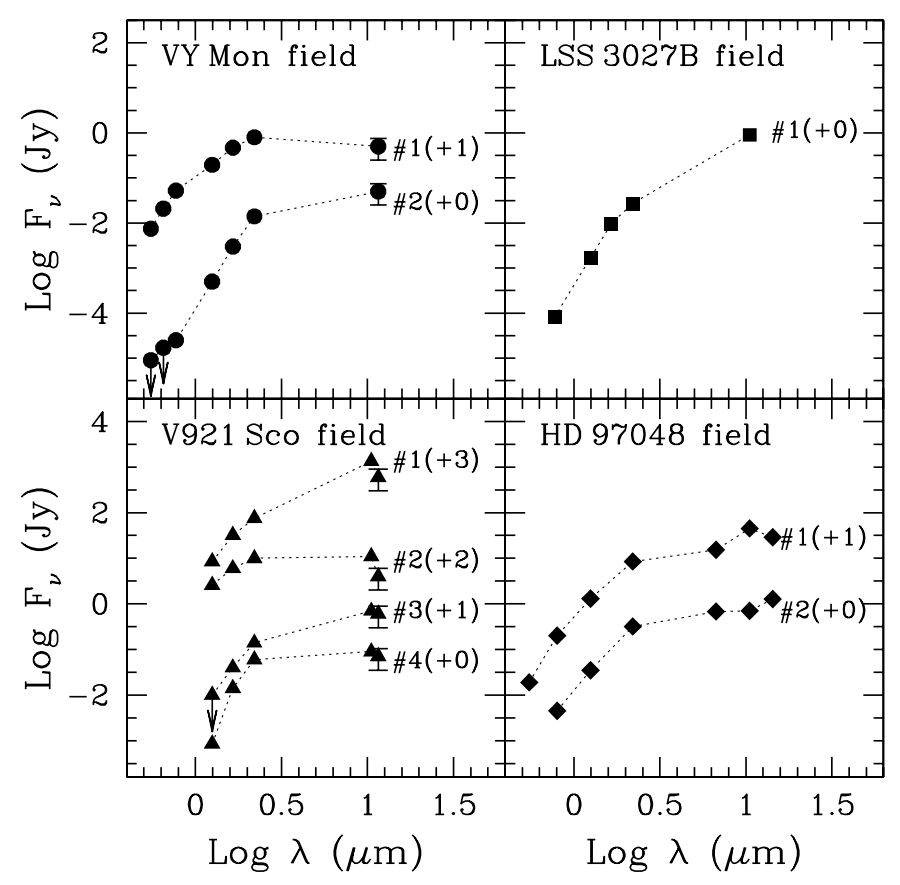

Fig. 1. Observed spectral energy distribution of the detected $10 \mu \mathrm{m}$ sources. Left top panel: VY Mon field; optical and mid-IR data from this paper, near-IR photometry from Testi et al. (1998). Left bottom panel: V921 Sco field; data from this paper. Right top panel: LSS 3027B field; data from this paper. Right bottom panel: HD 97048 field; optical and near-IR data from Persi et al. (2000) and Comerón et al. (1999); ISO data (6.7 and $14.3 \mu \mathrm{m})$ from Persi et al. (2000). Source names as in Table 2. The HD 97048 companions are HM 16 (\#1) and ISO-ChaI-126 (\# 2), respectively. When more than one source is plotted in a Panel, they have been shifted by the amount shown in parenthesis. Error bars, when not shown, are smaller than the size of the symbols.

The relatively low sensitivity of our observations prevents in practice any statistically significant measurement of the fraction of companions with/without disks around HAeBe stars, which was one of the original aims of this project. It is, nevertheless, clear that in several cases the HAeBe star is associated to other very young objects, probably the tip of the iceberg of a cluster of lower mass stars. It is therefore interesting to explore further the nature of the sources we detected.

The SEDs plotted in Fig. 1 show that all the $10 \mu \mathrm{m}$ companions are very red objects. This is seen clearly by their location on the color-color diagrams. Figures 4 and 5 plots the observed points in the $(J-H, H-K)$ and $(H-K, K-N)$ diagrams, respectively. Their location can be compared to the average colors of classical T Tauri stars (CTTS, Meyer et al. 1998) and of Herbig Ae stars (Hillenbrand et al. 1992). Note that while the locus of CTTS is rather well defined, that of Herbig Ae stars shows a larger dispersion. On both diagrams the location of the $10 \mu \mathrm{m}$ companions is roughly consistent with that of reddened pre-main sequence stars with circumstellar disks. The extinction $A_{V}$ derived from the near-IR color-color diagram appears to range from few to about $25 \mathrm{mag}$.

In the following section we will discuss the properties of the individual $10 \mu \mathrm{m}$ companions in detail, with the exception of the two companions of MWC 297, for which we have no information in addition to their $10 \mu \mathrm{m}$ fluxes. Also, we will not discuss the two sources associated to HD 97048, whose properties can be found in the literature (Persi et al. 2000 and references therein).

\section{Individual regions}

\subsection{V921 Sco}

The region around V921 Sco contains four $10 \mu \mathrm{m}$ sources. Their $J, H, K, N 2$ and $N 11.9$ fluxes are given in Tables 2 and 3; Fig. 1 shows their observed spectral energy distribution. All these sources are too weak in the optical and we were not able to obtain their optical spectra. Therefore, we cannot determine their spectral type nor their location on the HR diagram. We can, however, obtain some information on their nature from their near-infrared colors, using a statistical analysis that has been applied, for example, to embedded ISOCAM sources by Bontemps et al. (2001).

Firstly, we compute for each source the spectral index between 2.2 and $10 \mu \mathrm{m}\left(\alpha_{\mathrm{IR}}=\Delta \log \left(\lambda F_{\lambda}\right) / \Delta \log \lambda\right)$. We obtain negative values for three sources $(\# 2, \# 3$, \#4) and we 


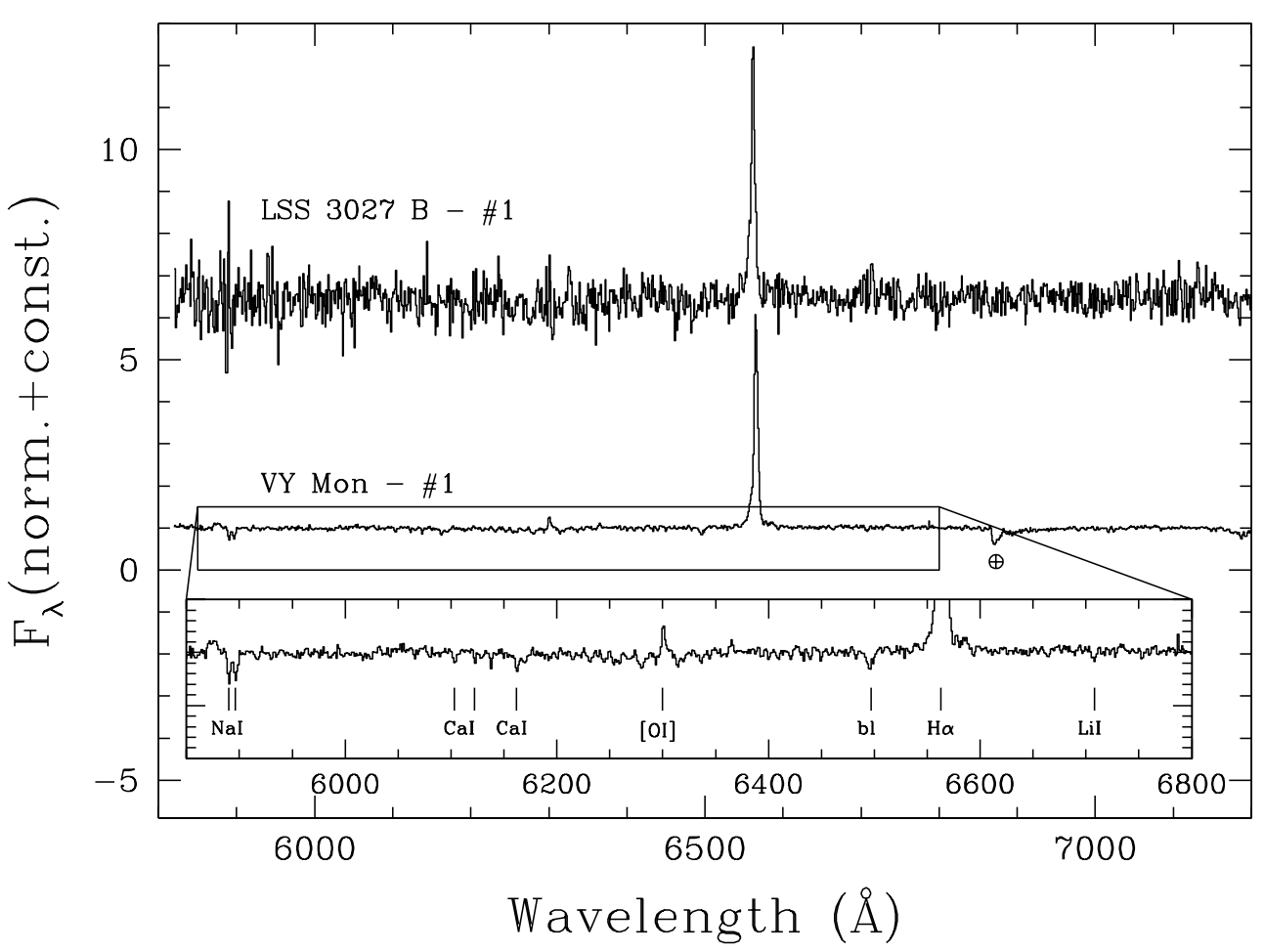

Fig. 2. Optical moderate resolution spectra of VY Mon-\#1 (bottom) and LSS 3027 B-\#1 (top). In the inset we show the absorption line identifications for VY Mon-\#1. The spectra have been normalised to the continuum and shifted vertically for clarity.

infer that they have an infrared SED similar to that of Class II ${ }^{1}$ objects Wilking et al. (1989) and of HAe stars (Hillenbrand et al. 1992). The nature of \#1 is more uncertain, as one can see from Fig. 1. The spectral index computed using the $K$ and $N 11.9$ magnitudes is $\alpha_{\mathrm{IR}} \sim 0.3$, while from the $K$ and $N 2$ magnitudes one derives $\alpha_{\mathrm{IR}} \sim 0.8$, typical of low-mass Class I or very embedded objects.

We estimate the extinction toward the four $10 \mu \mathrm{m}$ sources and their luminosity using their near-IR magnitudes and a statistical procedure developed by Meyer et al. (1997) (see also Bontemps et al. 2001). We adopt the reddening law determined by Cohen et al. (1981) for the standard CIT system:

$A_{V}=9.09 \times\left[(J-H)-(J-H)_{0}\right]$

$A_{V}=15.04 \times\left[(H-K)-(H-K)_{0}\right]$.

For the intrinsic colors, we have used the average values $(J-$ $H)_{0}=0.85$ and $(H-K)_{0}=0.55$ from the CTTS results of Meyer et al. (1997). As the dispersion in the intrinsic $(J-H)$ colors of CTTS is smaller, we have used the $(J-H)$ color excess when is it available, and the $(H-K)$ color excess otherwise. The location of the sources \#1, \#2, \#4 is shown in Fig. 4.

The dereddened $J$ (or $H$ ) magnitude can then be converted into an absolute $J$-band ( $H$-band) magnitude, $M_{J}\left(M_{H}\right)$, using the distance modulus of V921 Sco (9.51 for $d=800 \mathrm{pc}$ ) and the relation between the $J$ - and $H$-band magnitude and the stellar

${ }^{1}$ We note that the infrared SED classification scheme of Wilking et al. (1989) has not been devised for intermediate-mass stars and thus, in principle, it cannot be blindly applied to our infrared companions. luminosity $L_{\star}$ determined by Bontemps et al. (2001) for young CTTS:

$\log _{10}\left(L_{\star}\right)=1.49-0.466 \times M_{J}$

$\log _{10}\left(L_{\star}\right)=1.26-0.477 \times\left(M_{H}+0.2\right)$.

The derived values of $A_{V}$ and $L_{\star}$ are given in Table 4 . The uncertainties are very large, in particular for the most luminous \#4, for which the assumption of intrinsic colors typical of CTTS is likely to be inappropriate. We have therefore repeated the same procedure, using the analogous of Eqs. (1) and (3) derived for the sample of Herbig Ae stars of Hillenbrand et al. (1992) $\left((J-H)_{0}=0.98 ; \log _{10}\left(L_{\star}\right)=1.8-0.6 \times M_{J}\right)$. The results for \#4 are $A_{V}=22.5, M_{J}=-0.12, L_{\star}=75 L_{\odot}$, not very different from the values given in Table 4.

These results, even if significant only in a statistical sense, can be used to estimate the mass of the objects from a comparison with the evolutionary tracks on the HR diagram, assuming that they are coeval with V921 Sco. We give in Table 4 the values of mass and effective temperature corresponding to a V921 Sco age in the interval 0.1-1 Myr. All the four sources turn out to be intermediate mass objects, that once on the ZAMS will have spectral types A0-F0. This result confirms that at the distance and age of V921 Sco our TIMMI2 observations only detect discs around intermediate-mass stars.

\subsection{LSS 3027}

The star LSS 3027 is a young star of spectral type about B1 which powers a small HII region. It has distance $\sim 1800-1900 \mathrm{pc}$ and an extinction of $A_{V} \sim 1.6 \mathrm{mag}$ (Chu 1983). 


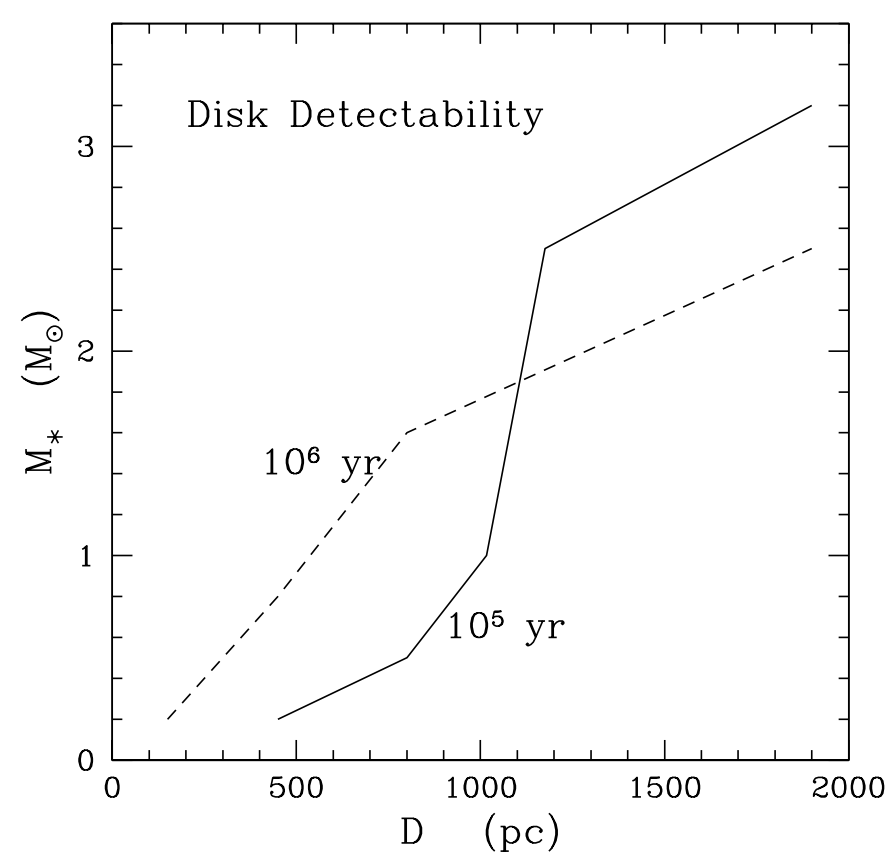

Fig. 3. Minimum stellar mass for the detectability of an irradiated disk as function of distance. The figure has been constructed by computing the $10 \mu \mathrm{m}$ flux of a flared disk irradiated by a star of given mass and age; we then plotted as function of distance the minimum stellar mass for which the predicted flux will be above the $3 \sigma$ limit of out TIMMI 2 observations. We have considered ages of $10^{5}$ and $10^{6} \mathrm{yr}$ (solid and dashed curves, respectively). For each mass, effective temperature and luminosity are taken from the Palla \& Stahler (1993) evolutionary tracks. Disk models are described in Natta et al. (2001).

With these parameters, it is located on the ZAMS in the HR diagram.

LSS 3027 was not included in our TIMMI2 field, which was centered on the Herbig Be star LSS 3027B, very likely at the same distance. This is a well known emission line star, of spectral type O8-B2 (Steenman \& The 1989). The extinction toward this object is subject of debate (see discussion in Porter et al. 1998); if we adopt $A_{V} \sim 6 \mathrm{mag}$ (as in Steenman \& The 1989), we obtain a luminosity of $2600 L_{\odot}$ for spectral type B2, and also LSS 3027B is close to the ZAMS.

Near LSS 3027B we detect a second source (\#1 in Table 2), whose spectral energy distribution is shown in Fig. 1. It is clearly a very red object, with $\alpha_{\mathrm{IR}} \sim 1.2$, typical of Class I sources. The optical spectrum (Fig. 2) is rather noisy, and does not show any feature, with the exception of strong $\mathrm{H}-\alpha$ in emission, as expected for a PMS star. Assuming as intrinsic colors the average near-infrared colors of CTTS, we obtain an extinction of about $16 \mathrm{mag}$ and a luminosity of about $75 L_{\odot}$. This is consistent with our detection limit for a region at $1800 \mathrm{pc}$ distance.

\subsection{VYMon}

We detected two $10 \mu \mathrm{m}$ sources associated to VY Mon. Source \#1 has a negative spectral slope $\left(\alpha_{\mathrm{IR}} \sim-1.3\right)$ and the location on the color-color diagrams typical of a moderately reddened Class II object. Using the same statistical procedure described for the objects near V921 Sco, we derive for an extinction $A_{V} \sim 4-5 \mathrm{mag}$ and a luminosity of about $8 L_{\odot}$.

These results can be checked further for object \#1, for which we have obtained also $V, R, i, J, H, K$ magnitudes (see Table 3 ) and an optical spectrum which is shown in Fig. 2. The $V, R, i$ magnitudes correspond to a star of spectral type about G0, reddened by $A_{V} \sim 4.7 \mathrm{mag}$. This is similar to the extinction toward VY Mon itself, and is much less than the extinction toward other $10 \mu \mathrm{m}$ sources, such as those near V921 Sco (see Sect. 5.1). The spectral type G0 is also consistent with the photospheric absorption features detected in our optical spectrum (see Sect. 3.2). At the distance of VY Mon, \#1 has then a luminosity of about $16 L_{\odot}$, and is a young star (age $<1 \mathrm{Myr}$ ) of about $2 M_{\odot}$. The strong $\mathrm{H} \alpha$ and [OI] emission and the tentative detection of LiI $\lambda 6708 \AA$ absorption confirm its youth. Note that the extinction is very similar to the statistical value obtained from the near-IR colors. The luminosity is a factor of $\sim 2$ higher. This is due to the fact that this particular source is unusually weak in $J$, even if no excess emission is present (see following). On the whole, given the uncertainties of the statistical method when applied to individual sources, this result is encouraging.

Figure 6 shows the SED of \#1, with the observed points dereddened by $A_{V}=4.7 \mathrm{mag}$. The two dotted lines show the emission of two black-bodies, one with $T=6000 \mathrm{~K}$, $L=16 L_{\odot}$, which shows the emission of the stellar photosphere, and one with $T=1300 \mathrm{~K}, L=4 L_{\odot}$, which approximately reproduces the infrared excess of the object. The solid line is the sum of the two. The infrared excess is about $15 \%$ of the stellar luminosity. Its properties (dependence on wavelength and luminosity) are not fit by standard disk models, either flat or flared. Data at longer wavelengths, and a good description of the region of the $10 \mu \mathrm{m}$ silicate feature, are required to speculate further on the exact nature of this source.

Source \#2 has a rather red spectrum, with a spectral index $\alpha_{\mathrm{IR}} \sim-0.2$. If we apply to it the statistical procedure appropriate for Class II sources, we derive a large extinction $\left(A_{V} \sim 15 \mathrm{mag}\right)$ but a rather low luminosity $\left(\sim 2 L_{\odot}\right)$ and mass (about $1 M_{\odot}$, assuming that it has an age of $1 \mathrm{Myr}$, i.e., that it is roughly coeval with \#1; the mass will be $<0.5 M_{\odot}$ for an age of $10^{5} \mathrm{yr}$ ). We do not expect a $10 \mu \mathrm{m}$ flux of about $0.05 \mathrm{Jy}$ from a Class II source of such luminosity at the VY Mon distance (see Fig. 7). It is possible that \#2 is an object in transition between Class I and Class II, and that we are underestimating its luminosity significantly.

\section{Discussion}

The properties of the $10 \mu \mathrm{m}$ sources are summarized in Table 4 . In spite of the large uncertainties, it is possible to see that in all cases, with the exception of the field around the nearby star HD 97048, we detected emission from intermediate-mass young stellar objects. Four of the objects have characteristics similar to those of CTTS or Herbig Ae stars, while two (possibly three) objects are probably closer to Class I objects.

A further check of these results can be obtained by comparing the observed $10 \mu \mathrm{m}$ fluxes to disk model predictions, similar to those presented in Fig. 3. Figure 7 shows that the 


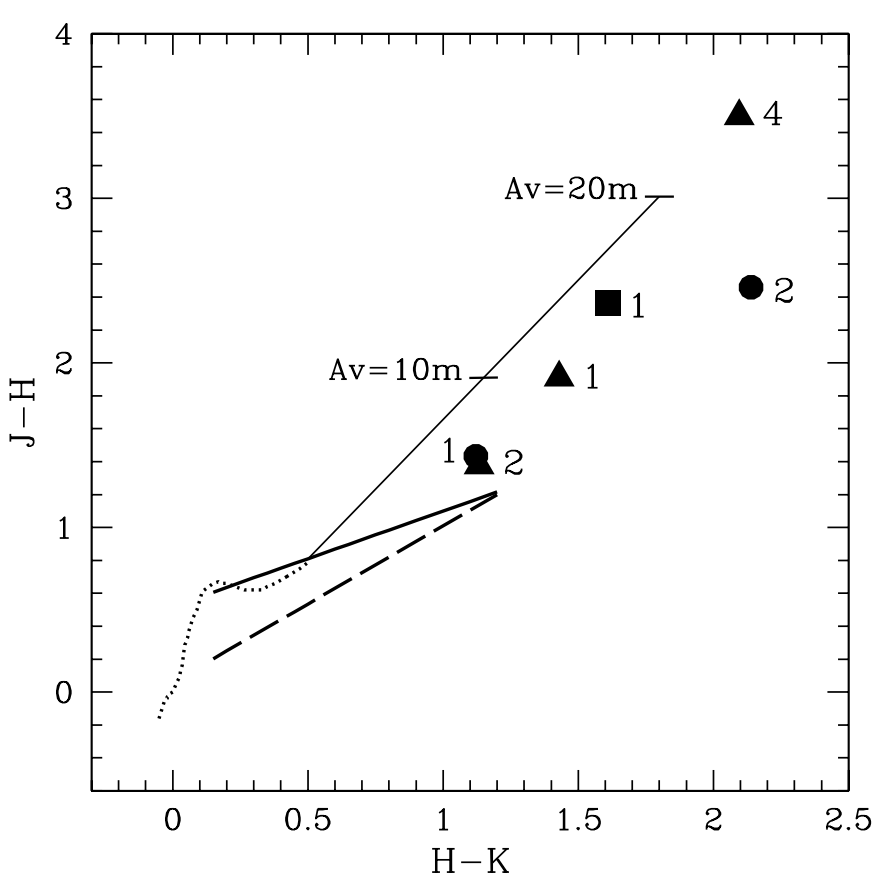

Fig. 4. $(J-H)$ versus $(H-K)$ diagram for the $10 \mu \mathrm{m}$ sources in the field of V921 Sco (triangles), LSS 3027 (square) and VY Mon (filled circles). The solid and dashed lines trace respectively a linear fit of the near-IR color distribution of CTTS (Meyer et al. 1997) and of Herbig Ae stars (Hillenbrand et al. 1992). Colors of MS stars (dotted line) are from Koornneef (1983) and Bessell et al. (1991); the slope of the interstellar reddening vector is from Cohen et al. (1981).

Table 4. Properties of the $10 \mu \mathrm{m}$ sources.

\begin{tabular}{lcccc}
\hline \hline Source & $\begin{array}{c}A_{V} \\
(\mathrm{mag})\end{array}$ & $\begin{array}{c}L_{\star} \\
\left(L_{\odot}\right)\end{array}$ & $\begin{array}{c}T_{\text {eff }} \\
(\mathrm{K})\end{array}$ & $\begin{array}{c}M_{\star} \\
\left(M_{\odot}\right)\end{array}$ \\
\hline V921 Sco field & & & & \\
$\# 1$ & 10 & 12 & 5000 & $1.5-2.5$ \\
$\# 2$ & 5 & 10 & 5000 & $1.5-2.5$ \\
$\# 3$ & 21 & 10 & 5000 & $1.5-2.5$ \\
$\# 4$ & 24 & 65 & $6000-8000$ & $3-3.5$ \\
LSS 3027B field & & & & \\
$\# 1$ & 16 & 75 & $6500-8500$ & $3-3.5$ \\
VY Mon field & & & & \\
$\# 1$ & 5 & 16 & 6000 & 2 \\
$\# 2$ & 15 & 2 & - & $0.5-1.5$ \\
HD 97048 field & & & & \\
$\# 1^{a}$ & 5 & 1.6 & 4000 & 0.7 \\
$\# 2^{a}$ & 4 & 0.3 & - & - \\
\hline
\end{tabular}

${ }^{a}$ From Persi et al. (2000), Comerón et al. (1999).

observations of V921 Sco-\#2,\#3,\#4 and of VY Mon-\#1 are consistent with the emission of disks heated by stars of mass of 1.5-3.5 $M_{\odot}$, as in Table 4. Sources V921 Sco-\#1, LSS 3027B$\# 1$, and VY Mon-\#2, which look like Class I objects, are stronger at $10 \mu \mathrm{m}$ than passive disk models predictions. This suggests that these objects may still be actively accreting.

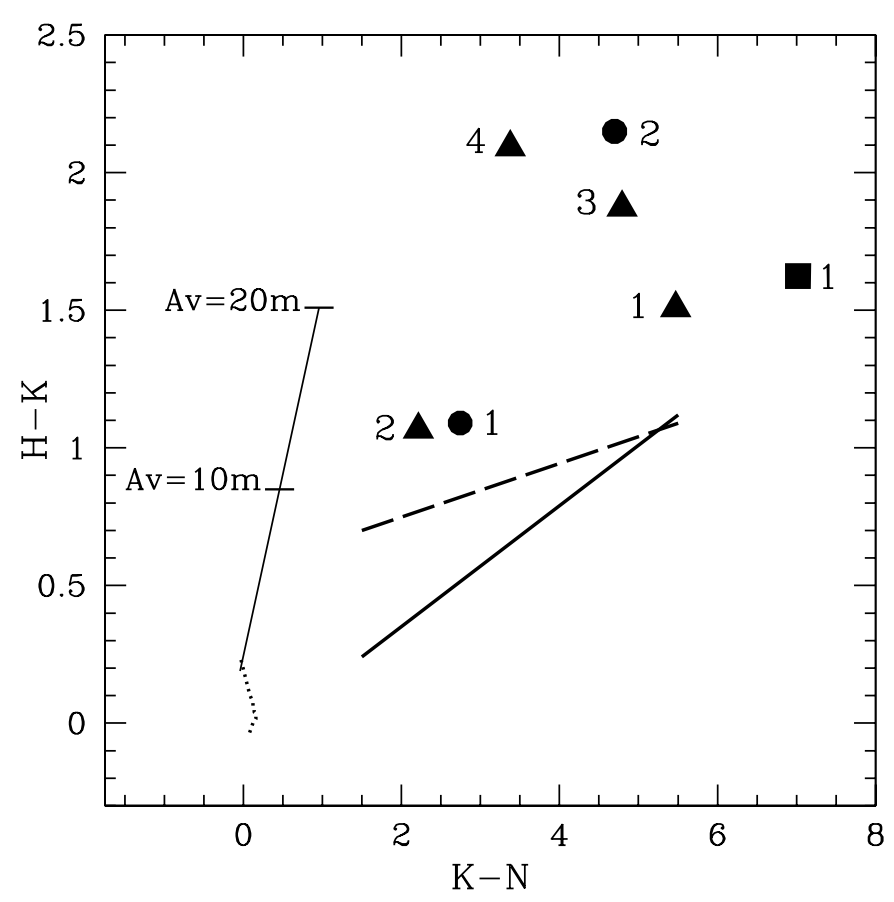

Fig. 5. $(H-K)$ versus $(K-N)$ diagram for the $10 \mu \mathrm{m}$ sources in the field of V921 Sco (triangles), LSS 3027 (square) and VY Mon (filled circles). The solid and dashed lines trace respectively a linear fit of the color distribution of CTTS in Taurus-Auriga (data from Beckwith, private communication) and of Herbig Ae stars (Hillenbrand et al. 1992). Colors of MS stars (dotted line) are from Bessell et al. (1991), the slope of the interstellar reddening vector from Rieke \& Lebofsky (1985). For consistency with the literature data on CTTS, HAe and MS stars, we estimated a $N$ magnitude for our sources from the fluxes observed in the narrow bands $N 2$ and $N 11.9$.

The detection of small clusters of highly reddened intermediate-mass stars associated to early Herbig Be stars has a number of implications. First of all, let us note that we do not detect any embedded source more massive than the optically visible Herbig star. Although we cannot exclude the possibility of even more embedded objects, that would not be visible at $10 \mu \mathrm{m}$ all the objects we detect are between 2 and 5 times less massive than the Herbig Be star. This confirms the assumption of Testi et al. (1999, Testi et al. (2002) that the optically visible Herbig $\mathrm{Be}$ is the most massive object in each cluster. Furthermore, this work shows that for studies of Herbig Ae/Be stars in lower spatial resolution far-infrared data the contamination radiation by companions is less than $\sim 3 \%$. In two fields, those around V921 Sco and TY CrA, we detected also diffuse mid-infrared emission with an extent of $\sim 10-20$ arcsec around the Herbig star. In these cases the low-resolution mid-infrared fluxes of the Herbig stars could be overestimated by as much as a factor $\sim 2$ in both V921 Sco and TY CrA.

The results of this paper also confirm that early Be Herbig stars are born within rather rich clusters, unless the IMF is very peculiar. Assuming a Scalo (1998) IMF, one would expect the ratio of the number of objects with mass in the range 1 to $4 M_{\odot}$ to the number of objects with mass in the range 0.1 to $1 M_{\odot}$ should be around $15-20 \%$. Considering the low number statistics and uncertainties, this fraction is consistent with the ratio of 


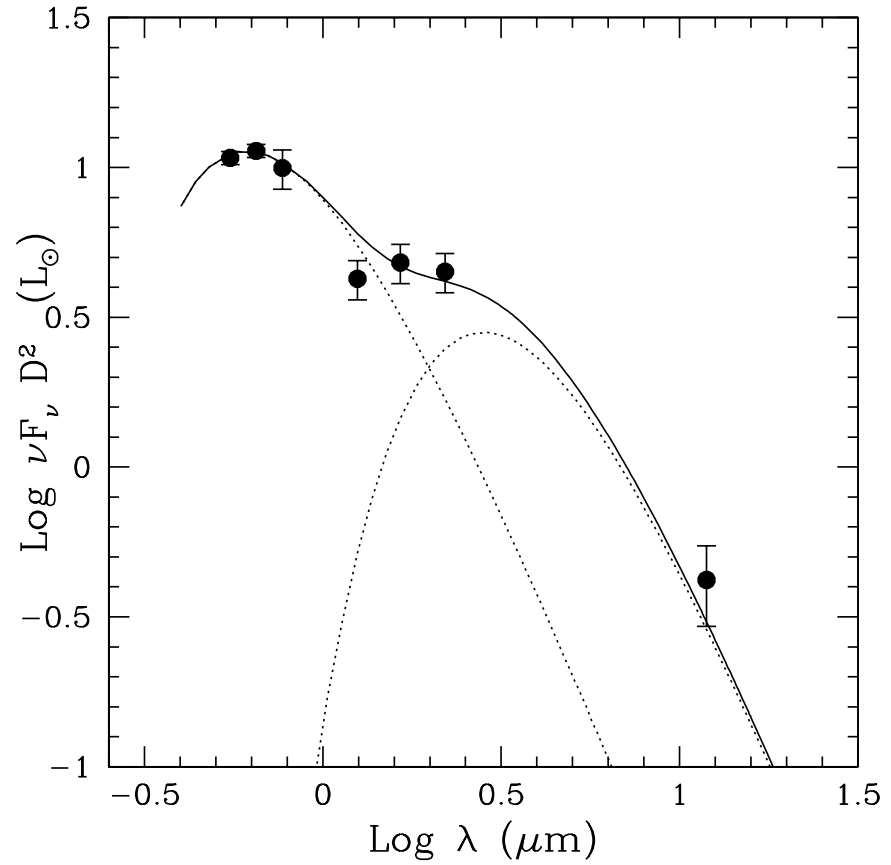

Fig. 6. Observed spectral energy distribution of VY Mon-\#1 dereddened by $A_{V}=4.7 \mathrm{mag}$ (dot points). The two dotted lines show the emission of two black-bodies, one with $T=6000 \mathrm{~K}, L=16 L_{\odot}$, which shows the emission of the stellar photosphere, and one with $T=1300 \mathrm{~K}, L=4 L_{\odot}$. The solid line is the sum of the two.

detected mid-infrared sources and the number of near-infrared companions ( $N_{\mathrm{m}}$ in Table 1$)$ covered by our survey. Also, the fact that at least some of these stars are highly embedded, suggests the presence of a rather dense molecular core in the region. It is possible that the parental cloud of the whole cluster has not been dispersed yet, or that the high extinction toward some of the sources is more local, due to an infalling envelope or a circumstellar cocoon. High resolution molecular observations of these regions would be of great interest.

Finally, these sources provide an interesting sample of sofar rare objects, namely embedded, very young intermediatemass stars. If we look, for example, at the list of stars later than A0 of Hillenbrand et al. (1992), none has $A_{V}>4$ mag, and most are significantly older than $10^{6} \mathrm{yr}$. Follow-up observations of our sample could improve our understanding of the early evolution of stars in the mass range $1.5-3.5 M_{\odot}$, of which little is known.

\section{Summary}

We have presented the results of a $10 \mu \mathrm{m}$ imaging survey of 12 fields around herbig AeBe stars obtained with TIMMI2 at the $3.6 \mathrm{~m}$ ESO telescope. The sensitivity of our observations was about $0.05 \mathrm{Jy}(3 \sigma)$; aside the Herbig Ae/Be stars, we detected sources in five fields. Of these, four are centered on early Be Herbig stars, while one is centered on the nearby A0 star HD 97048. This result is to be expected. First of all, only earlyBe stars have a (relatively) rich associated cluster (Testi et al. 1999). Secondly, with the exception of the very near-by fields, the sensitivity of our observations allowed detection only of intermediate-mass objects with $10 \mu \mathrm{m}$ excess emission.

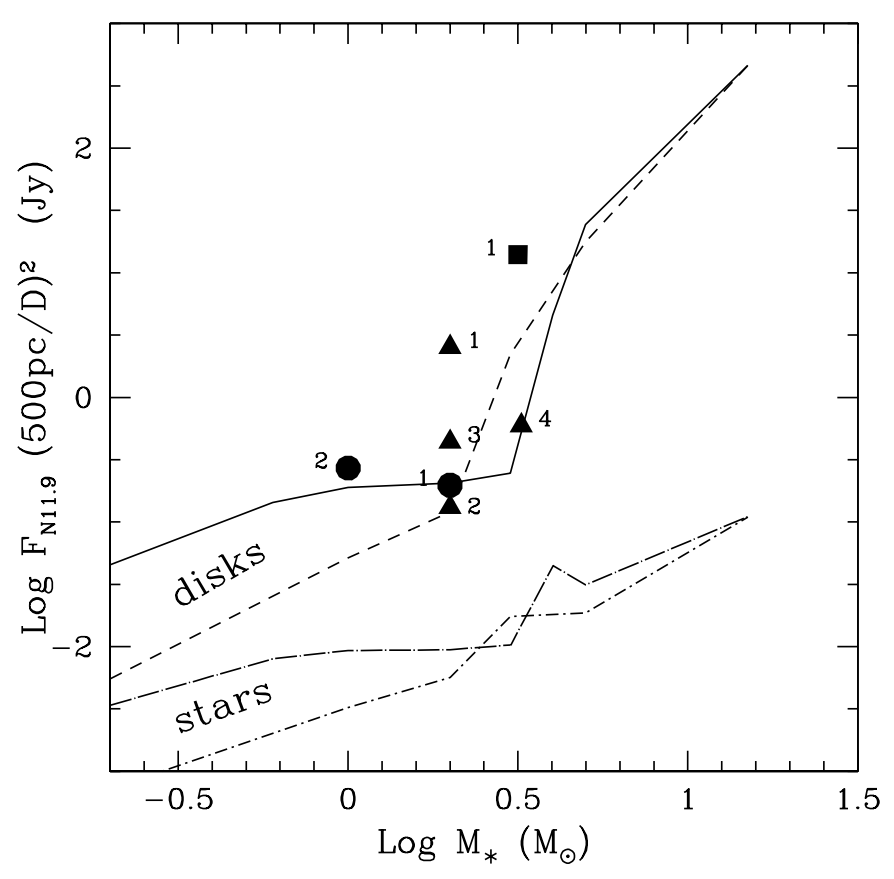

Fig. 7. Predicted flux in the $N 11.9$ band for irradiated disks as function of the mass of the central star. Solid and dashed curves show the predicted disk+ photosphere flux as function of the stellar mass for stars of age $10^{5}$ and $10^{6} \mathrm{yr}$, respectively. Long and short-dashed curves show the corresponding photospheric fluxes. The stellar parameters for a given mass and age are derived from the evolutionary tracks of Palla \& Stahler (1993). Disk models as in Fig. 3. The black points show the location of the $10 \mu \mathrm{m}$ sources corrected for reddening (see Table 4) and scaled to distance of $500 \mathrm{pc}$. Triangles refer to sources in the V921 Sco field, square to the LSS 3027B field (for which we have plotted the N2 flux), filled circles to the VY Mon field. We have assigned to VY Mon-\#2 a $1 M_{\odot}$ mass, corresponding to an age of $10^{6} \mathrm{yr}$; its location should therefore be compared to the dashed curve.

For several of the $10 \mu \mathrm{m}$ sources, we have supplementary optical and near-IR data. We have used this information to estimate the source parameters, such as extinction, luminosity and mass. In spite of the very large uncertainties, we find that, with the possible exception of VY Mon-\#2, they are heavily reddened $\left(A_{V}=5-24 \mathrm{mag}\right)$ intermediate-mass objects $(1.5-$ 3.5 $M_{\odot}$ ). In two, possibly three cases it looks as if one is observing deeply embedded, younger objects. The other detected sources are consistent with the emission of irradiated, passive optically thick disks, powered by the central star.

This sample of young, embedded intermediate-mass stars is unique so far. Follow-up observations are needed, first to confirm our identification and parameter estimates, then to study the early phases of the pre-main sequence evolution of such stars, which is still rather poorly known.

Acknowledgements. It is a pleasure to thank Nancy Ageorges and Michael Sterzik for the excellent service observing for one of our TIMMI2 runs. We also acknowledge the extensive and competent help of the ESO-La Silla support astronomers and telescope operators. This work was partly supported by ASI grant ARS 1/R/27/00 and ARS1/R/073/01 to the Osservatorio di Arcetri. 


\section{References}

Bessell, M. S., Brett, J. M., Scholz, M., \& Wood, P. R. 1991, A\&AS, 89,335

Bontemps, S., André, P., Kaas, A. A., et al. 2001, A\&A, 372, 173

Chu, Y. H. 1983, PASP, 95, 873

Cohen, J. G., Persson, S. E., Elias, J. H., \& Frogel, J. A. 1981, ApJ, 249,481

Comerón, F., Rieke, G. H., \& Neuhauser, R. 1999, A\&A, 343, 477

Fukugita, M., Ichikawa, T., Gunn, J. E., et al. 1996, ApJ, 111, 1748

Hamuy, M., Suntzeff, N. B., Heathcote, S. R., et al. 1994, PASP, 106, 566

Hillenbrand, L. A., Strom, S. E., Vrba, F. J., \& Keene, J. 1992, ApJ, 397, 613

Hunt, L. K., Mannucci, F., Testi, L., Migliorini, S., \& Stanga, R. M. 1998, ApJ, 115, 2594

Koornneef, J. 1983, A\&A, 128, 84

Landolt, A. U. 1992, ApJ, 104, 340

Meyer, M. R., Calvet, N., \& Hillenbrand, L. A. 1997, ApJ, 114, 288

Meyer, D. M., Jura, M., \& Cardelli, J. A. 1998, ApJ, 493, 222
Natta, A., Prusti, T., Neri, R., et al. 2001, A\&A, 371, 186

Palla, F., \& Stahler, S. W. 1993, ApJ, 418, 414

Persi, P., Marenzi, A. R., Olofsson, G., et al. 2000, A\&A, 357, 219

Persson, S. E., Murphy, D. C., Krzeminski, W., Roth, M., \& Rieke, M. J. 1998, ApJ, 116, 2475

Porter, J. M., Drew, J. E., \& Lumsden, S. L. 1998, A\&A, 332, 999

Rieke, G. H., \& Lebofsky, M. J. 1985, ApJ, 288, 618

Scalo, J. 1998, The imf revisited: A case for variations, in The Stellar Initial Mass Function, 201

Siebenmorgen, R., Prusti, T., Natta, A., \& Mulller, T. G. 2000, A\&A, 361,258

Steenman, H., \& The, P. S. 1989, Ap\&SS, 161, 75

Testi, L., Palla, F., \& Natta, A. 1998, A\&AS, 133, 81

Testi, L., Palla, F., \& Natta, A. 1999, A\&A, 342, 515

Testi, L., Palla, F., Natta, A., Vanzi, L., \& Zinnecker, H. 2002, in preparation

van Kerckhoven, C., Hony, S., Peeters, E., Tielens, A. G. G. M., \& Allamandola, L. J. 2000, A\&A, 357, 1013

Wilking, B. A., Lada, C. J., \& Young, E. T. 1989, ApJ, 340, 823 